\title{
THE GERONTOLOGICAL ASPECTS OF ATHEROMA
}

\section{AN APPROACH TO A PATHOLOGY OF SENESCENCE}

\author{
BY \\ ERICH GEIRINGER* \\ From the Edinburgh Gerontological Research Unit, Usher Institute, University of Edinburgh
}

We may, as a matter of convenience, distinguish two categories of gerontological research, the study of old age or old people on the one hand, and the study of ageing on the other. Almost the whole of pathology is our field in the study of old age; for whilst there are very few diseases which spare the aged, many present special features when occurring in them, either attacking them more readily or being more dangerous and less tractable. The study of these phenomena presents a vast and important task, in which, however, orthodox pathology can be of great help. In the study of ageing the field is much narrower and the guidance to be derived from existing disciplines much less.

Each one of the diseases of old age is characterized by the fact that it attacks individuals or groups of individuals, leaving a significant proportion of mankind untouched. These diseases are, however, only the superimposed complications of an underlying process of functional deterioration in which all human beings share, and it is with this universal process that we are concerned in the study of ageing.

It must be admitted that strict proof for the existence of such a separate process of ageing is lacking. The concept that we are all subject to an identical process or complex of processes of deterioration is based on purely circumstantial evidence and on an impression which has been prevailing in the human race throughout the centuries. But if, as a working hypothesis, we accept the existence of ageing as a separate physiological or pathological entity and begin to look round for its manifestations, we are struck by the extraordinary difficulty of finding them. In order to be acceptable as a part of the complex of senescence a phenomenon must fulfil at least two conditions: it must be exhibited by all the members of the species, and it must be demonstrable at a time at which the complications of old age are still rare.

Atheroma of the aorta is one of the very few

* Whaitt Research Scholar. conditions which not only passes the test of both? these criteria but also furnishes us with easily accessible and comparatively easily measurable $\stackrel{+}{-}$ lesions. An analysis of aortic atheroma might, $\underset{\sim}{\omega}$ therefore, apart from its intrinsic interest, contributeo to our understanding of the process of senescence.

\section{METHOD}

At autopsy the aorta was removed together with the iliac arteries and the main pulmonary trunk. It was $\overrightarrow{0}$ stored in 10 per cent. formol. At a later date it was divided into eight strips, in each of which the extent agod. severity of atheroma was graded according to an arbitrajy numerical scale. The arithmetic mean of the eight results constitutes the total grade of the vessel. At the same time a verbal description of the appearances was. written down. Each aorta is identified by a number命 only, and the grading was carried out in complete ignorance of the clinical and pathological findings in $\overline{\bar{\sigma}}$ the case. Bias was thereby excluded except perhaps in the few instances in which a peculiarity of the vesse called the circumstances of the case to mind. Equally, the classification of the clinical and pathological condi tions in each case was carried out without knowledge of the state of the aorta, and the two records were only brought together when both the grading of the vesse $B$. and the clinico-pathological classification of the case had been completed. The fact that all these investigations were carried out by one individual probably serves to enhance the consistency of the results.

The criteria used in determining whether hypertensions had been present were in the first place the clinical records and then the absolute and relative heart weight and the state of the renal arterioles. There are many sources of error in each one of these criteria, and not only were the whole of the other findings taken intov consideration (with the exception of course of the state of the aorta), but no case was classified as either a proveck hypertensive or a proved normotensive in which any onf of the above criteria allowed of any doubt. Whilst thi resulted in a low number of such classifications it is felt? that the increased reliability of the individual correlationso more than compensates for the loss in statistical signifi cance. 


\section{The Frequency of Atheromatous InVolvement}

A study, in this manner, of three hundred autopsies has confirmed the observation of Zinserling (1925) and others that atheroma of the human aorta becomes very frequent after the age of 6 months and is invariably present after the age of 8 years. In Fig. 1 which shows the extent of atheromatous involvement of 180 aortae, it can be seen that the zero-line is completely unoccupied.

This fact has never been given sufficient emphasis. To the histo-pathologist it cannot be of great importance; from a gerontological point of view, however, this 100 per cent. incidence (which has been confirmed in places as distant from one another as Switzerland (Saltykow), Russia (Zinserling), Germany (Lubarsch), and Scotland and at dates as widely separated as $1913,1919,1925$, and 1948) is highly significant. It entitles us to regard atheroma aortae as a racial characteristic of homo sapien's, a part, we might say, of the "normal pathology " of man.

\section{Pathogenesis of Atheroma}

The uncertainty which still prevails about the motor causes, the pathogenesis, of atheroma has lately tended to obscure the fact that we are perfectly familiar with its final exciting cause, which is the action of arterial blood pressure upon the aortic wall. This well substantiated fact is explicitly or implicitly recognized in all the important work in this field. To put the matter in a nutshell: the human aorta is neither adapted nor completely adaptable to the human aortic blood pressure.

From the time of onset of atherogenesis, and from other lines of evidence such as is offered by a study of atheroma of the pulmonary artery, we are even enabled to put an approximate value upon the critical pressure above which atheroma will make its appearance. It is about $50 \mathrm{~mm}$. $\mathrm{Hg}$. How pressure brings about this effect is an important question:

whether by dragging upon fixed points of the vessel wall (Duguid),

whether by traumatizing the intima and leading to intimal haemorrhage (Winternitz) or mural thrombosis (Duguid),

whether by causing a pressure ischaemia with subsequent degeneration of the intima (Moon),

whether by upsetting the colloid balance of

blood cholesterol (Anitschkow),

whether by the opening up, in the endothelium, of stomata through which an excess of bloodlipoids is passed (Leary, Lange), whether by producing shearing strains between vessel wall and blood stream (Allbutt),

whether by inducing intimal herniation (Krafka),

whether by weakening the media with subsequent dilatation and consequent compensatory proliferation (Thoma),

whether by loosening the ground substance and allowing the infiltration of plasma constituents (Virchow, Aschoff).

\section{The Pathogenic action of Normal Function}

These theories are, however, not relevant to our present purpose. They all have a common aetiological background, viz. the action of normal blood pressure upon the normal aortic wall. This is of considerable gerontological interest because it furnishes us with an example of the "pathogenetic action of normal function," of which we shall meet many more by and by in the study of ageing if the concept of senescence as a separate entity has any validity at all.

It is repugnant to our teleological instincts and to our Darwinian upbringing to look upon normal function as a pathogen. A glance at Fig. I helps us in this perplexity. The grades of atheromatous involvement are there characterized by the terms:

$\begin{array}{ll}\text { Minimal: } & \text { from } 0 \text { to } 1 \\ \text { Mild: } & \text { from } 1 \text { to } 2 \\ \text { Moderate: } & \text { from } 2 \text { to } 3 \\ \text { Marked: } & \text { from } 3 \text { to } 4 \\ \text { Severe: } & \text { from } 4 \text { to } 5\end{array}$

Atheromatous lesions of the first two grades are certainly without any clinical importance. It can be seen that atheroma does not, in the vast majority of cases, assume clinically significant proportions until well after the end of the natural period of reproduction. It does not, therefore, interfere with the survival of the species, and it does not, therefore, tend to become eliminated by natural selection. This, too, is a principle which may have a larger application, and I am tempted to suggest that an adequate definition of senescence might be that it is the sum-total of those inherited flaws in animal organization which become manifest only in the post-reproductive period and therefore show no tendency to become eliminated by natural selection.

\section{The SusceptibIlity of the Aorta to Atheroma}

Study of Fig. 1 also preserves us from the common mistake of trying to explain atheroma by the action of one single factor. The two main features which point to the presence of another factor or factors are first, the continued rise in the severity of atheroma 


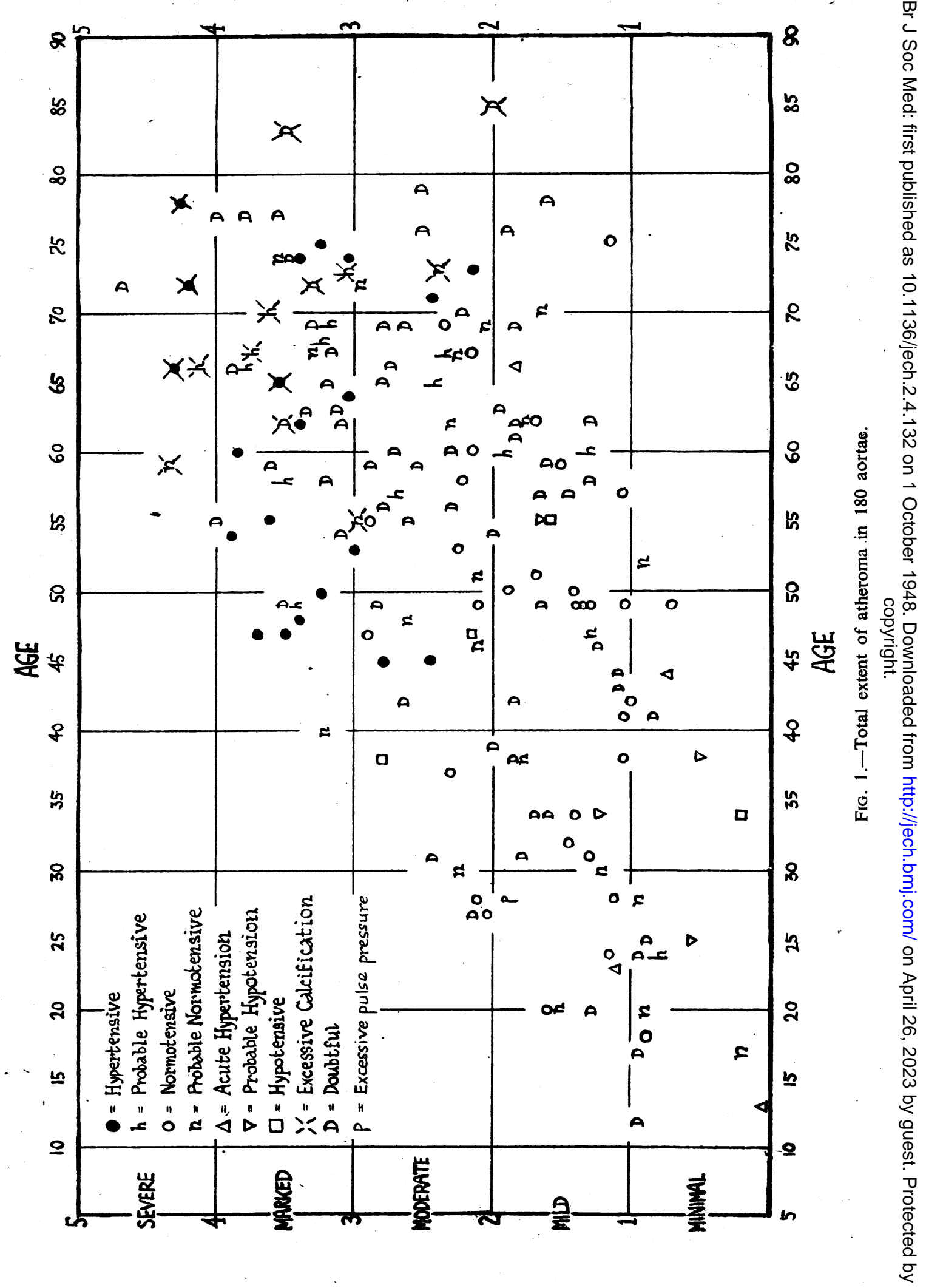


even in the most favourable cases after the time at which blood pressure becomes stabilized, and, secondly, the existence of scatter amongst normotensives of the same age. Any attempt to define these additional factors more closely would lead us at once into the pathogenetic battlefield, and we shall therefore be content to refer to these further causal conditions as " the susceptibility of the aorta to atheroma." We might then say that in the presence of a stable exciting cause the susceptibility of the aorta to atheroma increases gradually with mounting age and differs in different individuals at the same age. Recognition of these two factors allows us to form a rational idea of the nature of atheroma and resolves many of - the apparent paradoxes of this condition. Susceptibility may be normal or increased, pressure may be normal, increased, or decreased; and it is the combination in each individual case which determines the extent and severity of atheroma.

\section{The Behaviour of the Aortic Atheroma in Hypo-} TENSIVE CASES

Of particular interest in this connexion is the behaviour of aortic atheroma in hypotensive cases. At first thought we would expect abnormally low arterial pressure to be associated with an unusually mild degree of atheromatous involvement. This does in fact often obtain, as in the case of a woman of 34 (represented in Fig. 1) who had lingered on for two years, suffering from malignant cachexia and undergoing in that space of time two major operations (average blood pressure in the last three months of life: $98 / 68$; total extent of aortic atheroma: $0 \cdot 18$ ). In such cases we may see the result of the interaction of average aortic susceptibility with an exciting force of low intensity. More commonly the illness which induces hypotension interferes at the same time and to a commensurate degree with the reactivity and adaptability of the vascular parietes. Such a case, a woman of 47 (with chronic pulmonary tuberculosis and Addison's disease) is represented in Fig. 1. The extent of atheroma in these circumstances is about average for the age-group of the case. Lastly we have to consider conditions in which the interference with the reactivity of the tissues exceeds the hypotensive effect. Severe toxic diphtheria not uncommonly furnishes examples of this kind, and here extensive atheroma may be the result of a pressure considerably below normal. Such cases may be properly regarded as "relative hypertension," a concept of wide applicability in vascular pathology, the imperfect understanding of which has led in the past to the enunciation of the various "toxic" theories of the aetiology of atheroma.

Having taken due note of these three different types of hypotensive case we must in their analysis remember one further important detail, viz. that the hypotensive state is usually only a secondary one, obtaining often only for the last few months of life. Whilst we possess evidence suggesting that purely lipoid plaques may be resolved fairly quickly, it is obvious that fibrous lesions will still persist from the previous normotensive or hypertensive period. A striking confirmation that this last consideration is valid was obtained from a study of the verbal descriptions which are attached to each aortic grading. In describing the atheromatous lesions such terms as " purely lipoid," "predominantly lipoid," "fibro-lipoid" and "lipoid and fibrous" recur constantly. In two cases only the terms used were " almost entirely fibrous " and " mostly fibrous." Both these turned out to be cases of hypotension (a woman of 47 with Addison's disease, and a man of 55 with tuberculosis, mitral stenosis, and emphysema). This certainly suggests that hypotension may lead to an arrest in the new formation of atheromatous plaques whilst the old fibrous lesions persist (lipoid plaques already present undergoing either fibrosis or resorbtion). Unless this point is kept in mind a misleading picture of the relation between hypotension and atheroma might be arrived at.

\section{SeVERITY OF ATHEROMA}

In spite of this continuous rise as age advances, it can be seen from the base-line of Fig. 1, which represents the most favourable cases, and from the vast majority of the individual normotensive cases that, right up to the age of 80 , atheroma of the aorta remains a trivial complaint; an important fact, since the extent of aortic atheroma is a reliable index of the extent of atheroma in the coronary and in the cerebral vessels (Fig. 2). The hump of marked

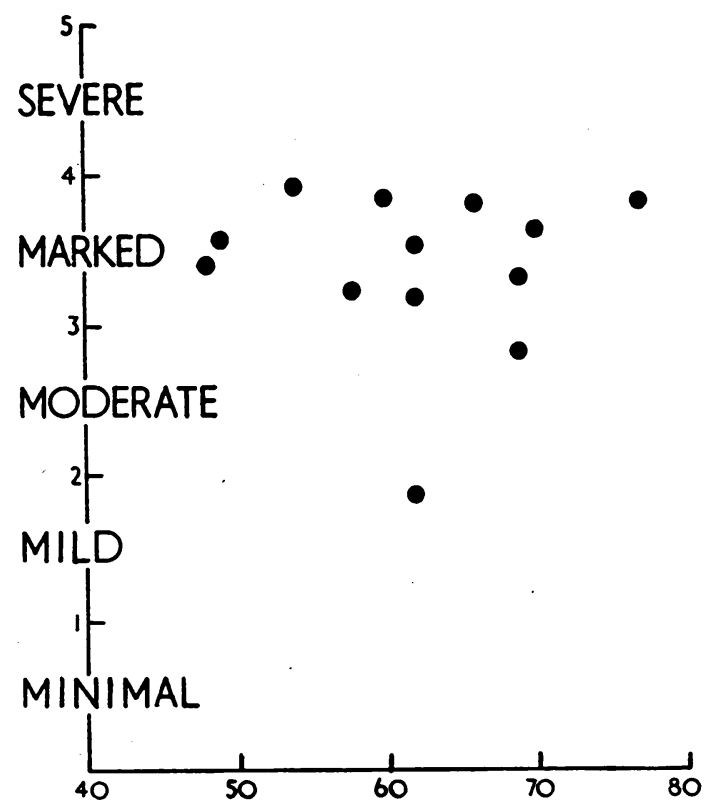

FIG. 2.-Extent of aortic atheroma in coronary thrombosis. 
and severe atheroma seen in Fig. 1 between the ages of 40 and 80 is largely made up of cases of hypertension, in other words of cases in which the normal age changes of the aorta have become unduly pronounced by the superimposition of a complicating factor. In this we may see a further characteristic of many of the changes of senescence. They are in themselves trivial and in no way threatening to life, but they form the basis on which dangerous complications arise. Ageing puts us at a disadvantage slight in itself but sufficient to tip the balance against us in our fight with our environmental and constitutional enemies.

If we were to prolong the base line to the right, a purely speculative exercise, we would see that atheroma need not reach a clinically significant level until the age of 140 and not a dangerous level until the age of about 170 . Of the complications which interrupt this peaceful ascent towards patriarchal heights we shall first consider hypertension.

\section{HYPERTENSION}

From whatever cause it may arise, hypertension almost invariably results in a great increase in the extent and severity of atheroma except when $\stackrel{C}{\circ}$ occurring before the age of 20 , when the adaptability 3 of the aortic wall is often great enough to prevent $\stackrel{\mathbb{Q}}{\stackrel{2}{2}}$ such an increase. In this series a very clear-cut division could be demonstrated between the aortic $\stackrel{\vec{\rho}}{\rightarrow}$ involvement in cases with normal blood pressure $\overline{0}$ and in those suffering from hypertension. Whilst the two groups share the region of moderate $\frac{\bar{c}}{\frac{5}{\sigma}}$ atheroma to some extent, no proved normotensive $\stackrel{\varnothing}{\triangle}$ was found in the region of marked or severe atheroma, and no proved hypertensive in the region of minimal or mild atheroma (Fig. 3). The correlation between severe arteriosclerosis and hypertension has so $\vec{\omega}$ impressed some authors that they consider hyper- $\rho$ tension to be the cause of atheroma (Moschcowitz, $\frac{\widehat{\phi}}{\circ}$ 1930). In view of the universal occurrence of atheroma from an early age, this conclusion is ${ }_{+}^{N}$ patently untenable unless indeed we widen the $\vec{\omega}$ meaning of the term "hypertension" to include $N$

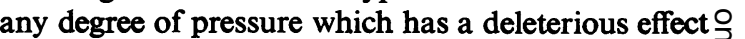
upon the arterial wall. Such a pressure, if withinthe normal limits for the age group of the case, is, $\bigcirc$ however, better referred to as a " relative hyper-? tension," a concept to which allusion has already $\underset{\Phi}{\mathbb{Q}}$

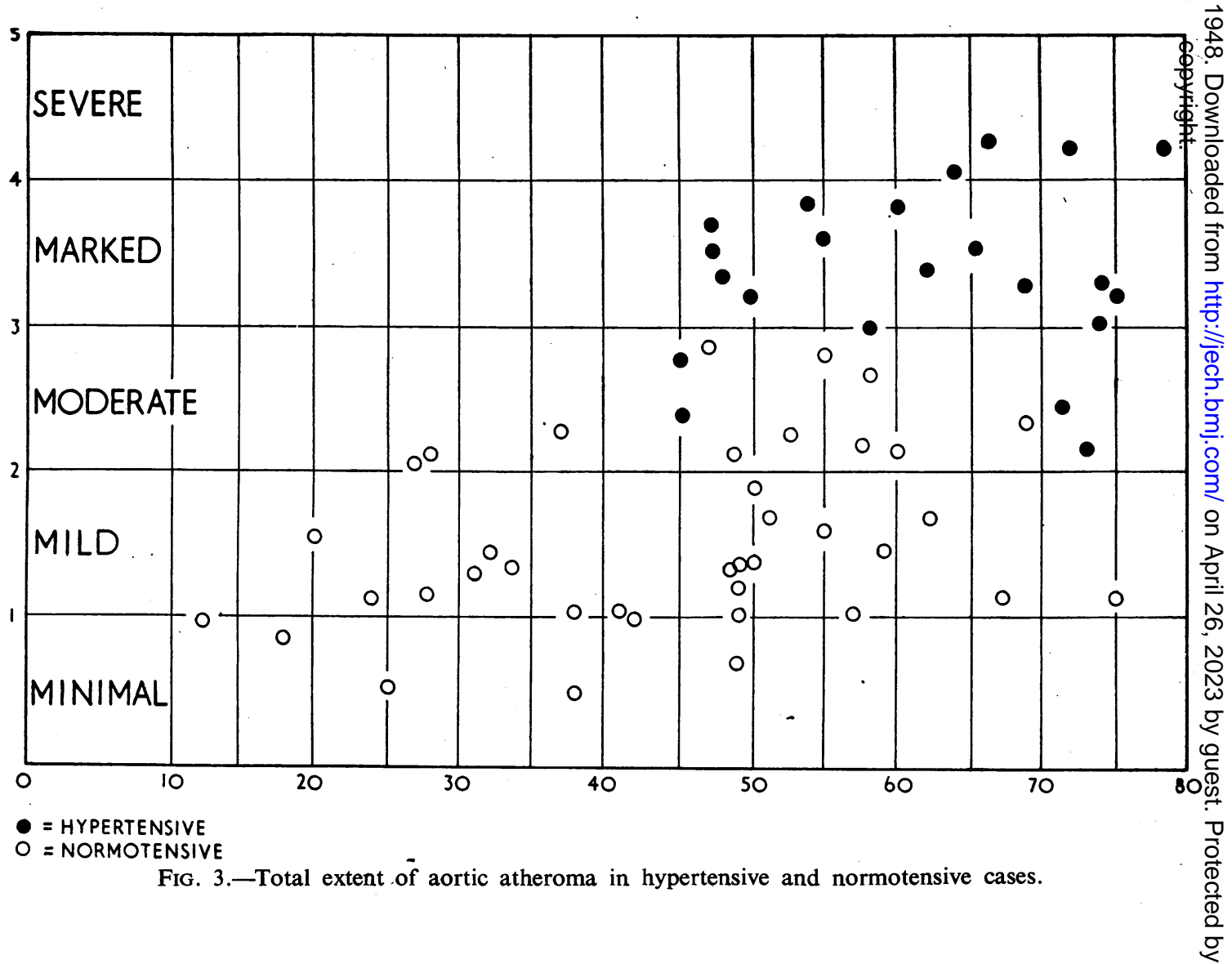


been made. Hypertension is a very frequent finding in diabetics, and it was therefore thought of interest to compare the degree of aortic atheroma in cases of diabetics with normal blood pressure with that of hypertensive diabetics. Fig. 4 shows that diabetics with normal blood pressure are no more severely affected than nondiabetic normotensive subjects. It would appear, therefore, that the frequent association of diabetes with severe atheroma is not a function of the diabetes itself but rather one of the hypertension which accompanies it so often and which even in the non-diabetic leads to an aggravation of atheroma.

\section{Calcification}

Whilst hypertension as a complication of atheroma is met with relatively early in life, there is another complication which transforms this benign manifestation of senescence into a dangerous disease and which is very infrequent before the age of 60 , and that is excessive calcification. Pathological collections of lipoid material are apt to have calcium deposited in them, and it would be very difficult indeed to find an aorta after the age of 30 in which microscopic examination would not reveal calcium in some at least of the more chronic plaques. In the majority of instances, however, these calcifications are discrete and the vessel remains pliable. From the age of 60 onwards there occur with increasing frequency cases of so-called " egg-shell aorta," in which excessive calcium deposition in the atheromatous lesions transforms wide stretches of the vessel into a rigid tube. That this is not simply the final stage in the natural evolution of atheroma is indicated by the fact that very extensive atheroma may be found at high ages without gross calcification. The natural end stage of atheroma is fibrosis, and the occurrence of excessive calcification in some cases points to the intervention in these cases of an additional factor.

Metastatic calcification may be induced experimentally with considerable ease by the administration of agents like vitamin $D$ or parathyroid hormone, and any tendency to metastatic calcification so induced will show its first effects in locations where lipoid accumulations or necrosis facilitate the deposition of calcium. We know also of various spontaneously occurring diseases which act in the

same way. Further, a halisteresis of the skeleton is common in old people and may be supposed to be caused by or be the cause of a disturbance of calcium metabolism.

It is therefore likely that, instead of looking upon cases of " egg-shell aorta" merely as cases of severely calcified atheroma, it might be more correct to see in them cases of atheroma complicated by some disturbance of calcium metabolism.

With this possibility in mind, the case notes of the fifteen aortae in which calcification had been graded either as marked or severe were subjected to a careful analysis. That ten of the fifteen are hypertensives is not surprising, since calcification of the extensive atheroma of hypertension is more likely to become graded as severe than the calcification of sporadic lesions. For the same reason the finding of twelve cases of nephrosclerosis among the fifteen is perhaps not startling, but we remember in this connexion the important role which the kidney plays in regulating calcium metabolism as an organ of excretion and, more important perhaps, as a stabilizer of the $p \mathrm{H}$ of the blood. Ten of the fifteen cases showed major pathology of the gastro-intestinal tract. Nine cases had evidence of metastatic calcification elsewhere, a very high proportion when it is remembered that this analysis was carried out in retrospect. The most striking finding, however, and one which was quite unexpected, was that no less than seven of these fifteen cases showed gross thyroid pathology: one case of myxoedema, one of past thyroidectomy for Graves's disease, two cystic nodular goitres, one smooth colloid goitre, and two cases with large cysts in one lobe-an 
incidence ten times that obtaining in the remainder of the present series. These fifteen cases thus form a group representing metastatic calcification plus renal, gastro-intestinal, and thyroid dysfunction in varying combination.

A clinico-pathological investigation is now in progress to elucidate the exact significance of these findings, but meanwhile they seem to underline the fact that cases of excessively calcified atheroma are not just cases that have progressed one stage further along the normal path of evolution of atheromatous lesions but are rather cases of atheroma plus a complicating factor.

A clear recognition of this relationship (with which probably few pathologists would be disposed to quarrel) is of considerable theoretical importance. As long as authors persist in classifying excessively calcified atheroma as " severe atheroma" they will continue to point to certain paradoxes which make an understanding of the nature of atheroma difficult. In Fig. 1, for example, we can see the case of a woman, 59 years old, in whom evidence points to the probability that her blood pressure was normal. She takes her place at the top of the chart surrounded entirely by hypertensives. Her case, regarded as one of severe atheroma, would tend to throw doubt on the correlation between height of blood pressure and severity of atheroma. Regarded as a case of atheroma with severe metastatic calcification superimposed, this difficulty vanishes.

But the realization that excessively calcified atheroma is complicated atheroma has also some practical importance, for this is a lesion which can often be diagnosed intra vitam by radiograph. It would seem important in handling such cases to remember that we are not dealing merely with an individual suffering from severe arteriosclerosis but that there is probably some upset of calcium metabolism as well.

These two major complications of atheroma are at the same time representative of two distinct types of condition which are met with in aged persons. Hypertension acts on the same function from which the basic change is derived and, by increasing the intensity of the exciting factor, leads to an aggravation of the atheroma and to its reaching prematurely a severity appropriate to a later age. We can readily think of parallel phenomena in other parts of the body. For example, the elasticity of lung tissue decreases with advancing age. There is, however, a group of individuals in whom this change occurs prematurely and to an exaggerated degree, leading to clinical emphysema in middle life. Such complications of ageing are, in their effect, pug ty quantitative ones.

Excessive calcification exemplifies the othe qualitative-type of complication. Here the basic condition is actually transformed in its nature by?an extraneous factor which interacts with it -in this instance by a disturbance of calcium metaboligm which transforms the lipoid plaque (which otherwise might go on to fibrosis) into a calcareous one. In terms of the aged lung this could perhaps be likered to infection which so readily supervenes in it transforming the senile emphysema into bronchonpneumonia.

\section{SUMMARY}

A study of three hundred aortae and a detaied analysis of 180 of them reveals the following poiruts:

1. Atheroma of the aorta is invariably present after the age of 8 . It is therefore to be regarded as a racial characteristic of mankind unless and untiDit can be shown to be due to an extraneous and ogly temporarily active factor.

2. Atheroma of the aorta is due to the effectof normal blood pressure on the normal aortic $\mathrm{w}_{\mathrm{oll}}$, thus furnishing an example of the pathogetetic action of normal function.

3. Clinically significant lesions do not as $\stackrel{\text { Rे }}{\text { inle }}$ occur before the age of 30, which explains how his defect in our organization has escaped the corrective action of natural selection.

4. Even after the age of 30 , uncomplicated atheroina is a mild and trivial condition, increasing only very gradually in severity as age advances.

5. It provides, however, a favourable soil on which complications arise in a great number of cases, transforming it from a harmless into a dangergus and often deadly disease.

6. The two major complicating conditions $\frac{0}{3}$ of atheroma are hypertension and excessive calcificationn.

In all these features we discern a distinctive pattern of pathological behaviour which I beligve to be characteristic of the phenomena of huningan senescence.

\section{REFERENCES}

Lubarsch. Quoted by Zinserling.

Moschcowitz, E. (1930). Amer. Heart J., 6, 171.

Saltykow. Quoted by Zinserling.

Zinserling, W. D. (1925). Virchows'Arch., 255, 67을 